\title{
ADVANCING NEXT-GENERATION PROTEOMICS: A POLYMER-PATTERNED MICROCHAMBER ENABLES INTEGRATION OF THE DISTINCT PROTEIN SEPARATIONS COMPRISING TWO-DIMENSIONAL ELECTROPHORESIS
}

\author{
Augusto M. Tentori, Alex J. Hughes, and Amy E. Herr \\ UC Berkeley/UCSF Joint Graduate Group in Bioengineering, Berkeley, CA, USA
}

\begin{abstract}
We introduce a new microfluidic approach for rapid, integrated two-dimensional electrophoresis (2DE), which uses a fundamentally different design strategy for integrating two the distinct assay stages of isoelectric focusing (IEF) and size-based electrophoretic separations. A novel device microchamber architecture coupled with spatially heterogeneous polymers enables the separated species from the $1^{\text {st }}$ dimension to be transferred for sizing separations without discretization into individual side channels. The carrier ampholyte based $\mathrm{pH}$ gradient is confined to the microchamber by incorporating immobilines into the polyacrylamide (PA) gel regions flanking the microchamber. The resulting $\mathrm{pH}$ is both linear and stable, with cathodic drifts $<3 \mu \mathrm{m} / \mathrm{min}$. Sample loading and focusing is rapidly achieved in $<30 \mathrm{~min}$. Species with isoelectric points ( $\mathrm{pI}$ ) of $<0.1 \mathrm{pH}$ units can be resolved and we estimate peak capacities of $\sim 100$ in the 1 st dimension. Protein transfer (enabled by ionic mobilization) and separation are rapid $(<10 \mathrm{~min})$ and preserve $1^{\text {st }}$ dimension separation information with band broadening factors $<1.3 \mathrm{x}$ and position drifts of $<50 \mu \mathrm{m}$.
\end{abstract}

\section{INTRODUCTION}

In biological processes, proteins are dynamic, ubiquitous conduits for transfer of information into action. Proteomic assays are central to the study of protein-mediated signaling. In particular, multidimensional protein separations enable the specific identification of protein species even amidst complex sample backgrounds.

Conventional 2DE integrates two serial assays to separate proteins by isoelectric point $(\mathrm{pI})$ and size [1]. Separating complex protein samples by IEF and protein sizing yields two physicochemical properties for each protein and enables the identification of species with a higher degree of precision then available from a single stage assay. For example, protein size separations are used for the identification of specific proteins; combined with charge separations, specific isoforms can also be identified [2]. Additionally, 2D separations provide better separation resolution and higher peak capacities compared to $1 \mathrm{D}$ assays, making 2D assays more suitable for the analysis of complex biological samples [3].

While powerful, conventional benchtop multidimensional separations are low throughput, semi-quantitative, and require manual intervention by a trained operator. Progress in proteomics is hindered by limited innovation in analytical technology.

Consequently, we introduce the development of a new microfluidic 2DE technology, as is critical to advancing the much needed 'proteomics revolution'. Unfortunately, conventional 2DE is labor and time intensive, making the assay a bottleneck for protein studies. Prior efforts to develop microfluidic $2 \mathrm{DE}$ have attempted to overcome these limitations, but suffer from information losses during transfer to the second stage. The as-ofyet unreported work we detail here introduces a fundamentally different design strategy for integrating two distinct assay stages. A novel device architecture (microchamber) coupled with spatially heterogeneous polymers overcomes key technical gaps that have plagued advances in on-chip proteomics.

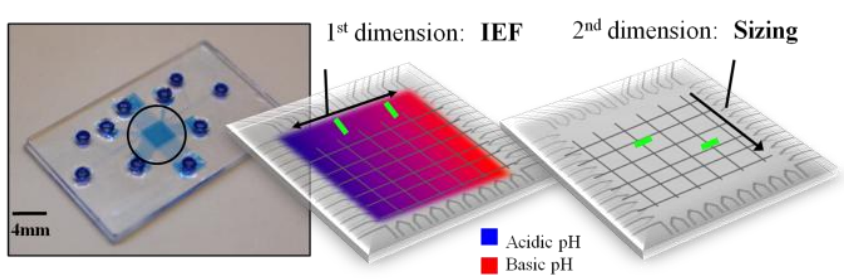

Figure 1: Polymer photopatterning in microchamber enables $2 D E$. Etched glass microdevice device features a $4 x 4 \mathrm{~mm}$ microchamber spanned by side channels for sample loading and electric field control. A multi-step PA photopolymerization protocol allows definition of spatially heterogeneous sieving media within the microchamber for IEF and subsequent size separations.

Established microfluidic 2DE employs intersecting microchannel networks supporting IEF and sizing. These integration efforts to unify $2 \mathrm{DE}$ in a microdevice have been severely limited owing to reliance on intersecting microchannel networks. In one implementation, species focused in a single channel are mobilized to a channel intersection using a low dispersion electroosmotic flow. When a band of interest reaches the intersection, the electric field direction is reversed to transfer individual bands to the second dimension [4]. In another incarnation species are focused in a channel flanked by multiple channels. The bands are then transferred to the array of channels for the second separation stage $[5,6]$.

In both cases, the continuous first stage assay is discretized in sampling to the second stage. Total peak capacity and $1^{\text {st }}$ dimension separation resolution depend on this sampling frequency in addition to the $1^{\text {st }}$ dimension separation performance. This general strategy for microfluidic 2DE leads to significant information losses that are not experienced with conventional benchtop approaches [7-9]. Thus, there is a critical need to introduce technology that combines the benefits microfluidic (speed, automation, low sample needs) and macroscale (lossless transfer) approaches.

Three major advances underpin our unique approach. Firstly, we introduce a new design utilizing microchamber and channel networks housing functional hydrogels (Figure 1). In contrast to previous studies, we use this approach to integrate multiple electrophoresis separations. Secondly, we utilize a novel photopatterning approach that allows spatial definition of physicochemical properties of hydrogels within this geometry. This marks a significant advance on state-of-the-art, as spatial definition of chemical properties has not been previously demonstrated. Thirdly, we demonstrate a novel integration approach that harnesses chemical mobilization of the first stage into distinct chamber regions, thus ensuring a transfer process with minimal losses between the two separation modes.

\section{METHODS AND RESULTS \\ Device Fabrication}

Etched glass chips were designed in-house using commercial computer-aided design (CAD) software. Designs were then sent to 
a commercial vendor (Caliper Life Sciences) for fabrication using well established glass wet-etching protocols. Subsequent customizations of the glass devices were all done in-house. PA gels within the microchannels of the device were photopolymerized using conventional UV photoinitiator (VA-086) and PA precursor and cross-linker reagents. Photopatterning was achieved using chrome photomasks in conjunction with an exposure system consisting of an inverted epifluorescence microscope coupled to a mercury lamp. Photopatterning protocols in conjunction with precursor solution wash steps enable the localization of the chemical and physical properties of the PA gel matrix with a high degree of spatial precision.

\section{pH Gradient Confinement}

A novel PA photopolymerization protocol allows us to confine the chemical conditions required for IEF within the microchamber (Figure 2). While achievable in a single channel, spatially confined IEF in a 2D geometry is a key component of our integration strategy and has never previously been reported. Chip designs that allow precise electric field control in 2D geometries have not allowed control over the conditions for the formation of the $\mathrm{pH}$ gradient in specific regions. Specifically, to achieve voltage shaping and therefore precise electrophoretic transport in a 2D geometry, side channel arrays flanking the main chamber are required $[10,11]$. These long control channels complicate the control of chemical conditions within the chamber by conventional approaches. This challenge was overcome by our novel fabrication approach. We achieve $\mathrm{pH}$ gradient confinement by using immobilized buffers to establish the basic and acidic boundary conditions flanking the microchamber. Immobilines are acrylamido weak acids and bases that can be incorporated into PA gels. Combinations of immobilines are used to achieve gels buffered at basic ( $\mathrm{pH} 9.3)$ and acid $(\mathrm{pH} 3.6)$ conditions.

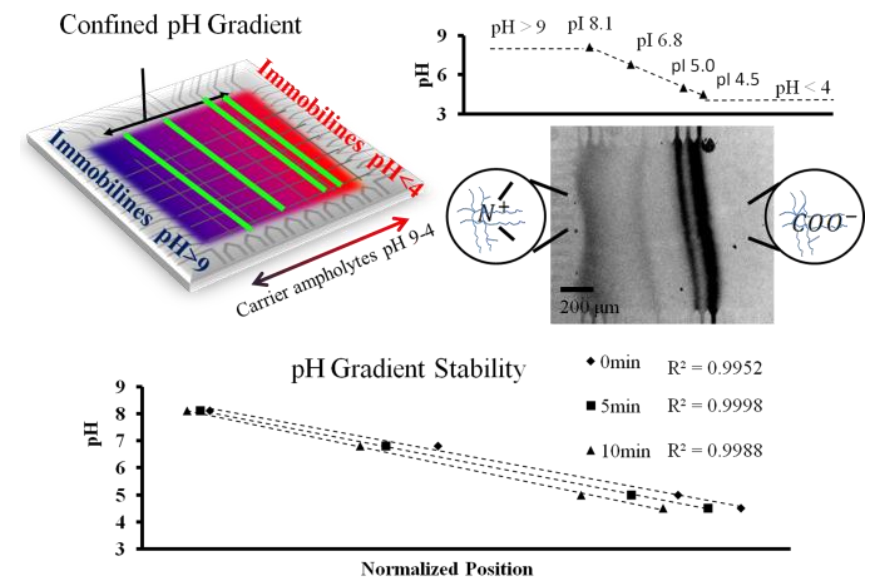

Figure 2: pH gradient confinement. Immobilines (acrylamido weak acid and bases) are incorporated into the gel flanking the microchamber. The presence of the stationary $\mathrm{pH}$ boundaries constrains the $p H$ gradient created by the (non-immobilized) carrier ampholytes within the chamber, demonstrated by the inverted micrograph of fluorescent UV pI markers performed in the $2 \mathrm{D}$ geometry. This $\mathrm{pH}$ gradient is stable with cathodic drifts $<3 \mu \mathrm{m} / \mathrm{min}$.

Gels containing (non-immobilized) polybuffer broad range (pH 4-9) carrier ampholytes are then patterned in the chamber. Upon application of an electric field, the carrier ampholytes migrate to their $\mathrm{pI}$. Ampholytes buffer at their $\mathrm{pI}$ and therefore using mixtures of several ampholytes with a large $\mathrm{pI}$ range, the $\mathrm{pH}$ gradient is established. Typically, the $\mathrm{pH}$ gradient for IEF using carrier ampholytes is formed by having a basic buffer (catholyte) and an acidic buffer (anolyte) at the terminals. In our approach, the stationary $\mathrm{pH}$ boundary conditions flanking the chamber constrain the $\mathrm{pH}$ gradient created by the carrier ampholytes within the chamber. $\mathrm{pH}$ gradients formed using our unique approach are stable with minimal cathodic drifts of $<3 \mu \mathrm{m} / \mathrm{min}$. Importantly, this approach enables highly flexible design, as the $\mathrm{pH}$ gradient range can be easily tuned for the desired application.

\section{Spatially Constrained IEF in 2D Geometry}

In order to integrate the chamber-based IEF with subsequent separations, a 1D "lane" for IEF in the chamber must be defined under the constrained $\mathrm{pH}$ gradient approach. Proper loading and precise voltage protocols are necessary to achieve focusing on a $1 \mathrm{D}$ lane in the chamber.

\section{A Confined GFP focusing in microchamber}

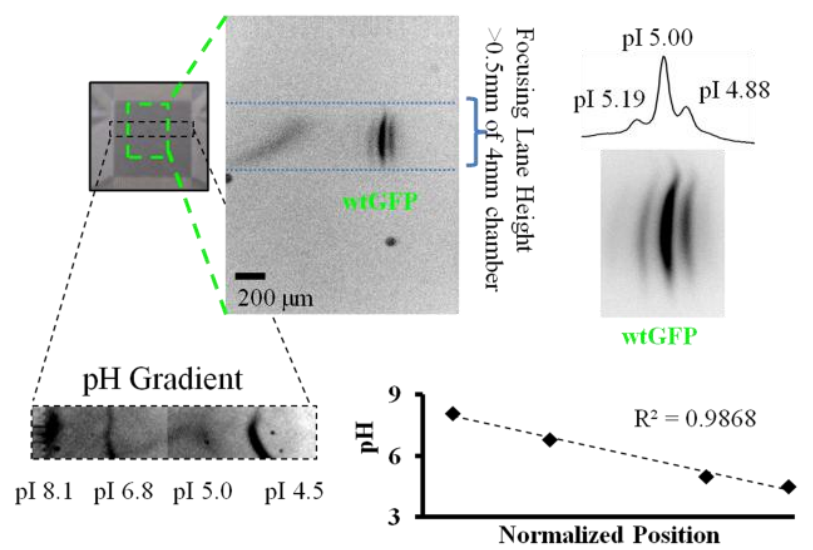

B Comparative GFP focusing in single channel
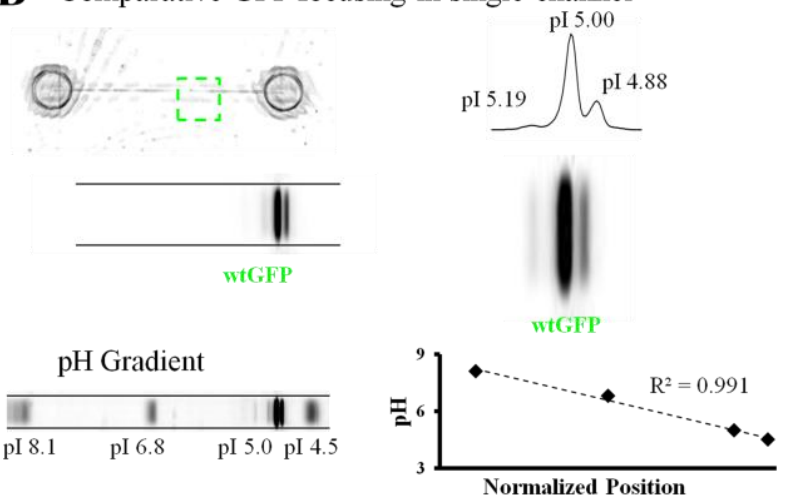

Figure 3: Confined high resolution and rapid IEF in microchamber. (A) Inverted fluorescence micrograph of wtGFP focusing in a confined ' $1 D$ lane' in the microchamber geometry. Sample loading is performed through a single side channel and electric field control minimizes dispersion. Stable, focused 3 isoform pattern of wtGFP (pIs 5.19, 5.00, 4.88) is visible <7min after loading into chamber. The $\mathrm{pH}$ gradient from 4-9 is linear and constrained within the microchamber. (B) For comparison, wtGFP focusing in a single $10.4 \mathrm{~mm}$ channel.

Confinement is achieved by loading the protein sample through a single channel and precise control of the electric field 
within the microchamber to prevent dispersion $[12,13]$. In the current design, samples are electrophoretically injected from a single channel in the cathode side. After the desired amount of material has been loaded, the loading well is washed with catholyte buffer. An additional benefit of our $\mathrm{pH}$ gradient confinement approach using immobilines is that ampholytes in entire chamber are uniformly focused. Without these uniform conditions, IEF on a 1D lane is not possible, as conductivity variations in focused and unfocused regions leads to substantial dispersion due to nonuniform electric fields.

Sample loading and focusing is rapid, taking less than $30 \mathrm{~min}$ to complete. Focusing occurs in a $<0.5 \mathrm{~mm}$ lane, thus enabling seamless integration with other regions of the microchamber. IEF performed in this configuration enables the separation of GFP isoforms resulting in resolution $<0.1 \mathrm{pH}$ units and peak capacities of $\sim 100$ in the first dimension (Figure 3). Further design improvements would include distinct wells for sample loading and catholyte reservoirs, thereby ensuring completely hands-free protocols. Resolving power on IEF systems depends on the steepness of the $\mathrm{pH}$ gradient and the separation axis length. The separation performance of this system can therefore be further enhanced by constraining narrower $\mathrm{pH}$ ranges and increasing the size of the microchamber.

\section{Transfer and Separation}

Low dispersion integration of the separate assay stages has been a hurdle to realizing high total assay performance. A major challenge is inherent to IEF, as proteins focused at their $\mathrm{pI}$ have no net charge and thus will not be mobilized unless the local $\mathrm{pH}$ is disrupted [14].

We initiate mobilization from the IEF stage to protein sizing by disrupting the $\mathrm{pH}$ gradient using electrophoretic introduction of a salt in the perpendicular direction to focusing (Figure 4). The introduction of an ionic species will cause the local hydronium ion and hydroxide ion levels to change in order to maintain the electroneutrality condition. Consequently, the local $\mathrm{pH}$ is altered allowing proteins to be mobilized given that they are no longer at their pI.

The mobilized proteins separate based on their relative mobilities. Protein transfer and separation is rapid, taking $<10 \mathrm{~min}$ to complete and preserves $1^{\text {st }}$ dimension separation information with band broadening of only $<1.3 x$ (competing microfluidic approaches result in effective band broadening of $\sim 10 \mathrm{x}$ due to undersampling). The bands drift $<50 \mu \mathrm{m}$ towards the acidic end during transfer, but retain their relative positions. Total assay time is $<1 \mathrm{hr}$.

The two key characteristics the ideal transfer mechanism would provide would be speed and transfer uniformity. A fast transfer will improve total assay performance as diffusion resulting from the stopping of the focusing electric field will be kept to a minimum. A uniform transfer will retain $1^{\text {st }}$ dimension separation information intact as well as enable size and electrophoretic mobility information to be extracted from the $2^{\text {nd }}$ separation. We are currently testing the uniformity of the transfer for broad $\mathrm{pH}$ ranges.

Another approach for mobilizing focused species is the introduction of an ionic detergent such as Sodium dodecyl sulfate (SDS) to impart a net charge on the proteins. We are currently testing how transfer performance will be affected with the introduction of ionic detergents compared to the demonstrated detergent-free transfer.

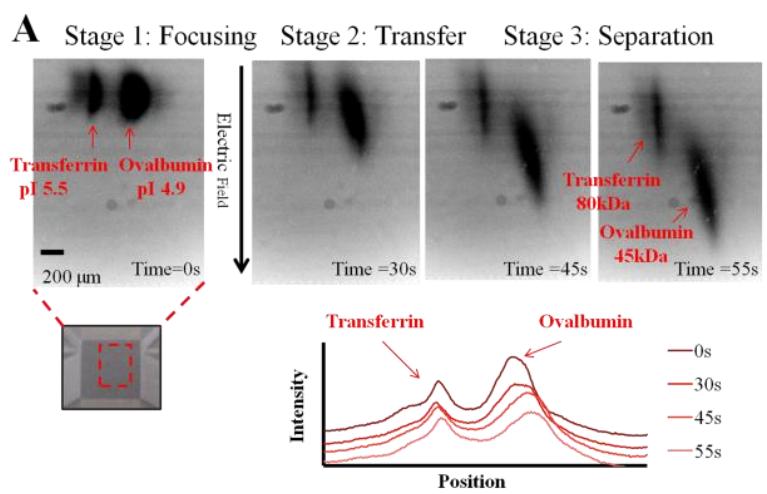

B Comparative single channel focusing

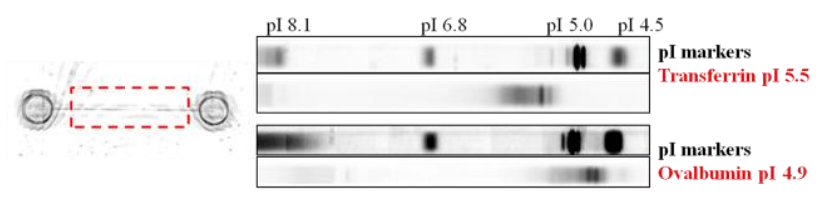

Figure 4: Microchamber integration enables lossless transfer for subsequent size separation. (A) Inverted fluorescence micrographs of CE540 labeled transferrin and ovalbumin focusing in confined '1D lane' in microchamber and subsequent transfer. Upon switching electric field direction and electrophoretic loading of a salt, the $\mathrm{pH}$ gradient is disrupted and the proteins migrate through the sieving matrix with mobilities proportional to their molecular weights. Offset intensity profile plots of transferrin and ovalbumin as they migrate through the chamber. Band broadening factors of $<1.3 x$ and position drifts $<50 \mu m$ are observed. (B) For comparison, transferrin and ovalbumin focusing in single channels.

\section{CONCLUSIONS}

Our approach combines the benefits of microfluidic (speed, automation, small volumes) and macroscale (lossless transfer) platforms. We are working towards developing capabilities for extracting both $\mathrm{pI}$ and molecular weight information from unlabeled biological samples. In addition, we hope to continue to improve total assay performance by further optimizing chip designs and experimental protocols. We see these advances as forming a new design paradigm for automated multi-stage assays with relevance spanning from fundamental biological inquiry to study of protein diagnostic biomarkers.

\section{ACKNOWLEDGEMENTS}

The authors gratefully acknowledge members and alumni of the Herr Laboratory at UC Berkeley for assistance and helpful discussions. The authors gratefully acknowledge financial support from the U.S. Department of Homeland Security's ORISE Fellowship (AMT) and the U.S. Department of Defense's NDSEG Fellowship (AJH). Partial conference travel support was generously provided by the Transducer Research Foundation (AMT). This work was funded in part by the National Science Foundation through CAREER Award (AEH, grant \# 1056035). $\mathrm{AEH}$ is an Alfred P. Sloan Foundation Research Fellow in chemistry.

This research was performed under an appointment to the Department of Homeland Security (DHS) Scholarship and Fellowship Program, administered by the Oak Ridge Institute for Science and Education (ORISE) through an interagency agreement between the US Department of Energy (DOE) and DHS. ORISE is 
managed by Oak Ridge Associated Universities (ORAU) under DOE contract number DE-AC05 06OR23100. All opinions expressed in this paper are the authors' and do not necessarily reflect the policies and views of DHS, DOE, or ORAU/ORISE.

\section{REFERENCES}

[1] Jung K., J. Reiche, A. Boehme, C. Stephan, S. A. Loening, D. Schnorr, W. Hoesel and P. Sinha (2004). "Analysis of subforms of free prostate-specific antigen in serum by twodimensional gel electrophoresis: potential to improve diagnosis of prostate cancer." Clin Chem 50(12): 2292-2301.

[2] O'Farrell, P. H. (1975). "High resolution two-dimensional electrophoresis of proteins." J Biol Chem 250(10): 40074021.

[3] Giddings, J. C. (1984). "Two-dimensional separations: concept and promise." Anal Chem 56(12): 1258A-1260A, 1262A, 1264A passim.

[4] Herr A. E., J. I. Molho, K. A. Drouvalakis, J. C. Mikkelsen, P. J. Utz, J. G. Santiago and T. W. Kenny (2003). "On-chip coupling of isoelectric focusing and free solution electrophoresis for multidimensional separations." Anal Chem 75(5): 1180-1187.

[5] Yang S., J. Liu, C. S. Lee and D. L. Devoe (2009). "Microfluidic 2-D PAGE using multifunctional in situ polyacrylamide gels and discontinuous buffers." Lab Chip 9(4): 592-599.

[6] Liu, J., C. F. Chen, S. Yang, C. C. Chang and D. L. Devoe (2010). "Mixed-mode electrokinetic and chromatographic peptide separations in a microvalve-integrated polymer chip." Lab Chip 10(16): 2122-2129.
[7] Li, X., D. R. Stoll and P. W. Carr (2009). "Equation for peak capacity estimation in two-dimensional liquid chromatography." Anal Chem 81(2): 845-850.

[8] Seeley, J. V. (2002). "Theoretical study of incomplete sampling of the first dimension in comprehensive twodimensional chromatography." J Chromatogr A 962(1-2): 2127.

[9] Davis, J. M., D. R. Stoll and P. W. Carr (2008). "Effect of first-dimension undersampling on effective peak capacity in comprehensive two-dimensional separations." Anal Chem 80(2): 461-473.

[10] Lerch, M. A. and S. C. Jacobson (2007). "Electrokinetic fluid control in two-dimensional planar microfluidic devices." Anal Chem 79(19): 7485-7491.

[11] Lerch, M. A., M. D. Hoffman and S. C. Jacobson (2008). "Influence of channel position on sample confinement in twodimensional planar microfluidic devices." Lab Chip 8(2): 316-322.

[12] He, M. and A. E. Herr (2009). "Microfluidic polyacrylamide gel electrophoresis with in situ immunoblotting for native protein analysis." Anal Chem 81(19): 8177-8184

[13] He, M. and A. E. Herr (2010). "Polyacrylamide gel photopatterning enables automated protein immunoblotting in a two-dimensional microdevice." J Am Chem Soc 132(8): 2512-2513.

[14] R. Rodriguez-Diaz, M. Zhu, T. Wehr (1997) "Strategies to improve performance of capillary isoelectric focusing." Journal of Chromatography A, 772 145-160

\section{CONTACT}

atentori@berkeley.edu, aeh@berkeley.edu

http://herrlab.berkeley.edu 